\title{
THE EFFECT OF DIFFERENT TECHNIQUES OF MACERATION-FERMENTATION ON THE PHENOLIC COMPOSITION OF RED WINES
}

\author{
Maria Iulia CERBU ${ }^{1, *}$, Valeriu V. COTEA ${ }^{1}$, Cătălin-Ioan ZAMFIR ${ }^{2}$, \\ Marius NICULAUA ${ }^{2}$, Ioana CĂLIN ${ }^{1}$, Andreea POPÎRDĂ ${ }^{1}$ \\ Cintia Lucia COLIBABA ${ }^{1}$, Ștefan TUDOSE SANDU-VILLE ${ }^{1}$ \\ *E-mail: cerbu.mariaiulia@yahoo.com
}

Received: Mar. 13, 2021. Revised: Mar. 25, 2021 Accepted: Mar. 28, 2021. Published online: Mar. 31, 2021

\begin{abstract}
Grapes, the quintessential quality factor in winemaking, are found in certain areas of the globe where viticulture thrives. The quality of wine products is directly influenced by the quality of the grapes, their process technology, the care and the quality of the premises and equipment used, as well as the conditions for the storage and use of the wines. In most red wine-making processes, it is preferred that the maceration process is accompanied by the fermentation process, as increase in the alcohol content favours the intensification of the extraction process. For this reason, both processes are combined in a single technological operation known as maceration-fermentation. The largest amount of polyphenolic compounds of wine, anthocyanins and tannins comes from the solid parts of the grapes - the skins and seeds, and these have a decisive influence on the phenolic character of wines. Maceration is a fractional
\end{abstract}

extraction which leads to the dissolution of the useful components of the grapes, which give the flavour, colour and taste typical of red wine. The aim of this research was to analyse the effect of different techniques of macerationfermentation on the phenolic composition of red wines obtained from Merlot, Pinot noir and Cabernet Sauvignon grape varieties in Copou-Iasi vineyard compared to red wines obtained from the same varieties in Murfaltar vineyard, located in the northeast and south of Romania, respectively. Wines obtained by maceration-fermentation in rotating tanks have higher values of the Folin-Ciocâlteu index (wines obtained from Pinot noir) in contrast to those obtained by the classical maceration-fermentation technique (wines obtained from Merlot and Cabernet Sauvignon).

Keywords: maceration; phenolic compounds; red wines; tannins; anthocyanins.

\footnotetext{
1 "Ion Ionescu de la Brad" University of Agricultural Sciences and Veterinary Medicine of lasi, 3rd Mihail Sadoveanu Alley, lași, Romania

${ }^{2}$ Research Centre for Oenology, 9 Mihail Sadoveanu, lași, Romania
} 


\section{Maria Iulia CERBU ET AL.}

\section{INTRODUCTION}

Wine is a complex mixture of hundreds of compounds, many of which are present in very low quantities, but play an important role in the evolution and consistency of the wine (Pereira et al., 2013; Rastija et al., 2009).

The alcoholic fermentation of musts in the presence of the solid sections of the berry (skins and seeds) produces red wines, whereas the fermentation of grape juice produces white wines (Ribéreau-Gayon et al., 2006).

Phenolic compounds contribute to the colour, taste and body of wines, and are thus important quality components. Polyphenols are extracted from grapes during the winemaking process, initially obtained by crushing of the fruit and intensified by the maceration. The most important classes of phenolic compounds are anthocyanins, tannins and flavones, which are specific groups of phenolic compounds, important for their sanogenic properties (Rodríguez-Delgado, 2002). They work together in order to provide a constant colour in wine, using pigmented polymers that form during different chemical processes (Cotea et al., 2000).

The purpose of macerationfermentation techniques is to facilitate the diffusion from the skin of large quantities of colouring substances in the must, which are insoluble in berry juice, but soluble in alcoholic solutions (must fermentation) when accompanied by an increase of temperature (Baiano et al., 2016).

In this paper, the evolution of the phenolic compounds content is studied, taking into account different techniques of macerationfermentation. The material used is grapes from Merlot, Pinot noir and Cabernet Sauvignon varieties from two vineyards: Copou-Iasi vineyard and Murfatlar vineyard.

\section{MATERIALS AND METHODS}

Grapes from Vitis vinifera varieties Merlot, Pinot noir and Cabernet Sauvignon, harvested in the Copou-Iasi (located in north-eastern Romania) and Murfatlar vineyards (located in southeastern Romania) were used to produce quality wines by different macerationfermentation techniques.

The climate of the Copou-Iasi vineyard is characterised by alternations of warm and quiet days with cold, frosty and windy days when passing from winter to spring and from autumn to winter. The climate is continental in Murfatlar vineyard, with hot and dry summers, moderate winters, early springs and late autumns, so that the growing period is the longest in the whole country.

Maceration is a complex process, biochemical and biophysical in nature at the same time, and an operation with deep oenological implications. For a certain period of time two phases of must remain together, in order to extract from the skins and other solids parts primarily phenolic compounds (anthocyanin pigments and tannins), which essentially express the character and general appearance of red wines (Cotea et al., 2000).

Grape samples and winemaking. Cabernet Sauvignon, Merlot and Pinot 


\section{THE EFFECT OF MACERATION-FERMENTATION ON THE PHENOLIC COMPOSITION OF RED WINES}

noir grapes were harvested from the Copou-Iasi and Murfatlar vineyards and experimental samples were obtained. In the experimental wine processing facility of the University of Agricultural Sciences and Veterinary Medicine (USAMV) in Iasi, a classical maceration-fermentation in three variants (V1, V3, V5) was applied, while in the Murfatlar cellars maceration-fermentation was done in rotating tanks also in three variants (V2, V4, V6), as follows: V1 CS - Iasi Cabernet Sauvignon; V2 CS - Murfatlar Cabernet Sauvignon; V3 M - Iasi Merlot; V4 M - Murfatlar Merlot; V5 PN - Iasi Pinot noir; V6 PN - Murfatlar Pinot noir.

The evolution to phenolic compounds content is studied, taking into account the different techniques of maceration-fermentation. We applied two types of maceration-fermentation to highlight the different phenolic potential of the wines depending on the different viticultural area of the vineyards and the different climatic conditions.

\section{Study of phenolic compounds and colour characteristics of red wines}

The total polyphenols index is expressed by the optical density (absorbance) analysed at the wavelength of $280 \mathrm{~nm}$ and expresses the content of phenolic compounds (phenolic acids, tannins and colouring compounds). In order to know the content of phenolic compounds in wines, the following were determined: the values of the total polyphenol index (TPI), the FolinCiocalteu index (FCI) and the total anthocyanins content. The TPI values make it possible to appreciate the wines according to their suppleness, namely: TPI $<30$ denotes supple wines to taste, TPI $=$ 30 50, well-formed wines with full and vigorous taste, and TPI $>50$ denotes astringent wines, with excess polyphenols (Waterhouse et al., 2016).
The Folin-Ciocâlteu Index (FCI) describes the phenolic compounds with reducing proprieties and is based on the oxidation in a basic medium of the phenolic compounds from wine by the Folin-Ciocâlteu reagent. The blue colouration is proportional to the total amount of phenolic compounds and has a maximum absorption about $750 \mathrm{~nm}$ (visible light) (OIV-MA-AS2-10).

The principle of the method for determining the total anthocyanins, or the $\mathrm{D} 280$ index, is based on the strong absorption of ultraviolet light by benzenic cores, characteristic of phenolic compounds, reaching a maximum absorption at the wavelength $\lambda=275 \sim 280$ $\mathrm{nm}$, and expresses the total content of the phenolic compounds of wine (tannins, anthocyanins, flavones etc.)

The determination of the chromatic characteristics of the wines under study was performed one year after they were obtained, according to STAS 6182/35-75, by the usual OIV spectrophotometric method. The chromatic parameters CIELAB $76\left(\mathrm{~L}^{*}, \mathrm{a}^{*}, \mathrm{~b}^{*}\right.$, intensity of colour $\left(\mathrm{C}^{*}{ }_{a b}\right)$ and tone $\left.\mathrm{h}_{a b}\right)$ were obtained by calculation for each experimental variant, as well as the computerised simulation of the wine colour (Schwedt, 1997; GarcíaGuzmán et al., 2015).

\section{RESULTS AND DISCUSSION}

In order to evaluate the content of phenolic compounds in the present wines, the total polyphenol index, the Folin-Ciocâlteu index, as well as the total content of polyphenols and the total content of anthocyanins were determined. The values of the analysed parameters obtained for the six proposed experimental variants are given in Table 1. 
For the three varieties of grapes, the data obtained show that the value of the total polyphenols index varies depending on the macerationfermentation process applied. Thus, for the Cabernet Sauvignon variety, the values are between $45.65 \sim 54.82$, for Merlot between 39.17 58.55, and for the Pinot noir between 40.94 52.01.

Table 1 - Total polyphenols index, Folin-Ciocalteu index, total polyphenols content and total anthocyanins content of Cabernet Sauvignon, Merlot and Pinot noir wines obtained by different maceration-fermentation processes

\begin{tabular}{ccccc}
\hline $\begin{array}{c}\text { Experimental } \\
\text { samples }\end{array}$ & TPI & FCl & $\begin{array}{c}\text { Total polyphenols } \\
\text { content (mg GAE/L) }\end{array}$ & $\begin{array}{c}\text { Total anthocyanins } \\
\text { content (mg GAE/L) }\end{array}$ \\
\hline V1 - CS & 45.65 & 38.83 & 2779.8 & 341.3 \\
\hline V2 - CS & 54.82 & 44.43 & 3338.2 & 323.84 \\
\hline V3 - M & 39.17 & 32.52 & 2385.21 & 391.8 \\
\hline V4 - M & 58.55 & 38.75 & 3565.34 & 274.18 \\
\hline V5 - PN & 40.94 & 33.98 & 2493 & 351.7 \\
\hline V6 - PN & 52.01 & 38.47 & 3167.09 & 279.78 \\
\hline
\end{tabular}

The variants with which the classical maceration-fermentation technique was used showed the lowest values of the total polyphenols index, respectively 39.17 for Merlot wine, 40.94 for Pinot noir wine and 45.65 for Cabernet Sauvignon wine. By comparison, the values for the wines obtained by the macerationfermentation process in rotating tanks, the values are between 52.01 for Pinot noir and 58.55 for Merlot.

Therefore, we can say the Cabernet Sauvignon wine obtained by classical maceration-fermentation technique can achieve aproximately the same amont of FCI as Merlot and Pinot noir wine obtained by maceration-fermentation in rotative tanks.

In order to be able to evaluate the content of wines in phenolic compounds and to compare the quantities in which the two main components are found - anthocyanins and tannins - the total amount of phenolic compounds, expressed in $\mathrm{mg}$ $\mathrm{GAE} / \mathrm{L}$ and the total content in anthocyanins, expressed in $\mathrm{mg} \mathrm{GAE} / \mathrm{L}$, were determined (Table 1).

The anthocyanins content is different depending on the variety, the conditions of the harvest year and the maceration technique used. Thus, it is observed in figure 1 that in the case of wines obtained by classical maceration-fermentation technique, they have a higher amount of anthocyanins than those obtained by maceration-fermentation in rotative tanks technique, these being between $341.3 \sim 391.8 \mathrm{mg}$ GAE/L and respectively, the content of anthocyanins between $274.18 \sim 323.84$ $\mathrm{mg}$ GAE/L. The highest values were obtained for wine V3 - M with 391.8 $\mathrm{mg} \mathrm{GAE} / \mathrm{L}$ and the lowest values for wine of the same variety V4 - M, but different technique, with $274.18 \mathrm{mg}$ GAE/L. 


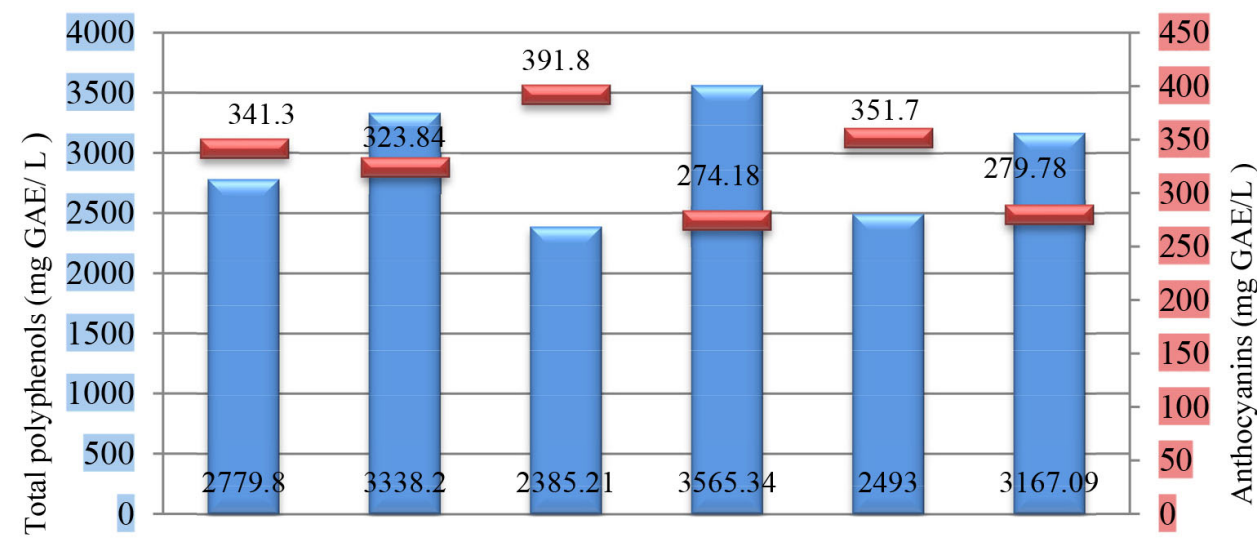

$\mathrm{CS}-\mathrm{V} 1 \quad \mathrm{CS}-\mathrm{V} 2 \quad \mathrm{PN}-\mathrm{V} 3 \quad \mathrm{PN}-\mathrm{V} 4 \quad \mathrm{M}-\mathrm{V} 5 \quad \mathrm{M}-\mathrm{V} 6$

Figure 1 - Anthocyanins content (mg/L) and total content of phenolics compounds of Cabernet Sauvignon (CS), Merlot (M) and Pinot noir (PN) wines

In the case of wines obtained from grapes belonging to the Merlot variety, the colours of the V3 - M and V4 - M variants are very close. For the Pinot noir wines obtained by the variants of classic macerationfermentation and macerationfermentation in rotating tanks, from the computerized colour simulation it appears that V6 - PN has a more intense colour than V5 - PN.

The chromatic parameters CIELAB 76 obtained by calculation for each experimental variant Table 2., as well as the computerised simulation of the wine colour, are shown in.

Table 2 - Selected chromatic parameters CIE-LAB 76 for wines samples

\begin{tabular}{cccccc}
\hline Variant & $\begin{array}{c}\text { Clarity } \\
\text { (L) }\end{array}$ & $\begin{array}{c}\text { Chromaticity } \\
\text { (green -a, } \\
\text { red +a) }\end{array}$ & $\begin{array}{c}\text { Chromaticity } \\
\text { (blue -b, } \\
\text { yellow +b) }\end{array}$ & $\begin{array}{c}\text { Tone } \\
\text { (H) }\end{array}$ & $\begin{array}{c}\text { Computer } \\
\text { simulation of wine } \\
\text { colour }\end{array}$ \\
\hline $\mathrm{CS}-\mathrm{V} 1$ & 7.8532 & 37.8763 & 12.9629 & 0.6059 & \\
\hline $\mathrm{CS}-\mathrm{V} 2$ & 7.1097 & 36.9878 & 12.9054 & 0.6061 & \\
\hline $\mathrm{M}-\mathrm{V} 3$ & 6.7083 & 35.9151 & 11.5440 & 0.6057 & \\
\hline $\mathrm{M}-\mathrm{V} 4$ & 6.7046 & 35.8859 & 11.5393 & 0.6019 & \\
\hline $\mathrm{PN}-\mathrm{V} 5$ & 9.7491 & 40.2097 & 16.7137 & 0.6032 & \\
\hline $\mathrm{PN}-\mathrm{V} 6$ & 9.3998 & 39.8771 & 13.9224 & 0.6009 & \\
\hline
\end{tabular}

The chromatic characteristics of the wines can vary in great intervals, which is due to the different years of harvesting, the vineyards from which the raw material is taken, the varieties and, last but not least, the technology applied to the grape processing.
Computer simulation of the wine colour was possible using the DIGITAL COLOR ATLAS 3.0 program in order to highlight colour differences and wine classification. This allows an easier understanding of the differences between the chromatic 
parameters obtained by calculation and makes it possible to differentiate and classify wines visually. Thus, the most intensely coloured wines were obtained in the case of the first two variants (V1 - CS and V2 - CS) processed through the two variants of maceration-fermentation (i.e., classic maceration-fermentation and maceration-fermentation in rotating tanks).

\section{CONCLUSION}

This research confirms that the different maceration-fermentation techniques contribute positively to some modifications in the phenolic composition, colour and sensory characteristics of red wines.

The anthocyanins content of wines is different depending on the maceration technique used. The results obtained vary within large limits from $274.18 \mathrm{mg} \mathrm{GAE} / \mathrm{L}$ (V4 - M) when the maceration was done in rotating tanks, to $391.8 \mathrm{mg} \mathrm{GAE} / \mathrm{L}(\mathrm{V} 3-\mathrm{M})$ when the wines were obtained by classical maceration-fermentation. In the variants obtained in rotating tanks, the anthocyanins content ranged between $274.18 \mathrm{mg}$ GAE/L (V4 - M) and $323.84 \mathrm{mg}$ GAE/L (V2 - CS), while between $341.3 \mathrm{mg} \mathrm{GAE} / \mathrm{L}$ (V1 - CS) and $391.8 \mathrm{mg} \mathrm{GAE} / \mathrm{L}(\mathrm{V} 3-\mathrm{M})$ in those obtained by classical maceration-fermentation.

Also, the data obtained showed that the value of the total polyphenol index varies depending on the maceration-fermentation process applied. For the wines obtained by the maceration-fermentation in rotating tanks, the highest values of the total polyphenols index (TPI) were obtained with values between 52.01 (Pinot noir) and 58.55 (Merlot), in contrast with the wines obtained by classic maceration-fermentation that recorded values of 39.17 (Merlot) and 45.65 (Cabernet Sauvignon).

The determination of the chromatic characteristics reflected that the most intensely coloured wines were obtained in the case of wines made from Cabernet Sauvignon grapes by both maceration-fermentation methods, but with the observation that the wine obtained by the macerationfermentation in rotating tanks was more intensely coloured than that obtained by the classical macerationfermentation method.

\section{REFERENCES}

Baiano Antonietta, Scrocco Carmela, Sepielli Grazia \& Del Nobile, M.A. (2016). Wine processing: A critical review of physical, chemical, and sensory implications of innovative vinification procedures. Crit. Rev. Food Sci. Nutr., 56(14): 2391-2407, DOI: 10408398.2013 .842886

Cotea, V.D., Pomohaci, N., Nămoloşanu, I., Stoian, V., Popa, A., Sîrghi C. \& Gheorghiţă, M. (2000). Oenology - Grape processing and wine production. (in Romanian), Vol. I, Ceres Publishing House, Bucharest.

García-Guzmán, J.J., Hernández-Artiga, M.P., Palacios-Ponce de León, L. \& Bellido-Milla, D. (2005). Selective methods for polyphenols and sulphur dioxide determination in 


\section{THE EFFECT OF MACERATION-FERMENTATION ON THE PHENOLIC COMPOSITION OF RED WINES}

wines. Food Chem., 182: 47-54, DOI: 10.1016/j.foodchem.2015.02.101

Pereira, V., Albuquerque, F., Cacho, J. \& Marques, J.-C. (2013). Polyphenols, antioxidant potential and color of fortified wines during accelerated ageing: The Madeira wine case study., Molecules, 18(3): 2997-3017, DOI: $10.3390 /$ molecules 18032997

Rastija, V., Srečnik, G. \& Medić-Šarić, M. (2009). Polyphenolic composition of Croatian wines with different geographical origins. Food Chem., 115(1): 54-60, DOI: 10.1016/j.food chem.2008.11.071

Ribéreau-Gayon, P., Glories, Y., Maujean, A. \& Dubourdieu, D. (Eds.) (2006). Handbook of enology, Vol. II - The Chemistry of Wine. Stabilization and Treatments. 2nd
Edition., John Wiley \& Sons, West Sussex.

Rodríguez-Delgado, M-Á., GonzalezHernandez, G., Conde-Gonzalez, J.-E. \& Perez-Trujillo, J.-P. (2002). Principal component analysis of the polyphenol content in young red wines. Food Chem., 78(4): 523-532, DOI: $\quad 10.1016 / \mathrm{s} 0308-8146(02) 002$ 06-6

Schwedt G. (1997). The essential guide to analytical chemistry. Brooks Haderlie Publishing House, Chichester, USA, pp. 16-17, New York.

Waterhouse, L.A., Sacks, G.L. \& Jeffrey, D.W. (2016). Understanding wine chemistry. 1st Edition, John Wiley and Sons, Chichester, 470 pp., DOI: 10.1002/anie.201700489. 